This is the submitted version of the following article:

Quesada-González D., Baiocco A., Martos A.A., de la Escosura-Muñiz A., Palleschi G., Merkoçi A.. Iridium oxide (IV) nanoparticle-based electrocatalytic detection of PBDE. Biosensors and Bioelectronics, (2019). 127. : 150 - . 10.1016/j.bios. 2018.11.050,

which has been published in final form at https://dx.doi.org/10.1016/j.bios.2018.11.050 () https://dx. doi.org/10.1016/j.bios. 2018.11.050. This manuscript version is made available under the CC-BY-NC-ND 4.0 license http: // creativecommons. org/licenses/by-nc-nd/4.0/ 


\title{
Iridium oxide (IV) nanoparticle-based electrocatalytic detection of PBDE
}

\author{
Daniel Quesada-González ${ }^{\mathrm{a}}$, Alessandra Baiocco ${ }^{\mathrm{a}, \mathrm{b}}$, Andrea A. Martos ${ }^{\mathrm{a}}$, Alfredo de la \\ Escosura-Muñiz ${ }^{\mathrm{a}}$, Giuseppe Palleschi ${ }^{\mathrm{b}}$, Arben Merkoçia, ${ }^{\mathrm{a} *}$ \\ ${ }^{a}$ Nanobioelectronics \& Biosensors Group, Institut Català de Nanociència i Nanotecnologia (ICN2), CSIC and The Barcelona Institute of \\ Science and Technology (BIST), Campus UAB, 08193, Bellaterra, Barcelona, Spain. \\ ${ }^{b}$ Dipartamento di Scienze e Technologie Chimiche, Università di Roma, Tor Vergata, Rome, Italy. \\ 'Institució Catalana de Recerca i Estudis Avançats (ICREA), P. Lluís Companys 23, 08010 Barcelona, Spain. \\ *arben.merkoci@icn2.cat
}

\begin{abstract}
Polybrominated diphenyl ethers (PBDEs) are a type of flame retardants which are currently banned in EU and USA due their hazardousness for humans and mammals. However, these compounds were highly used during more than 30 years and still persist in the environment since they are resistant to degradation. Herein we present a biosensor for the detection of PBDEs using screen printed carbon electrodes (SPCEs) based on the electrochemical monitoring of water oxidation reaction (WOR) catalyzed by iridium oxide (IV) nanoparticles (IrO2 NPs). Our assay shows a limit of detection of 21.5 ppb of PBDE in distilled water. We believe that such an IrO2 NPs-based electrocatalytic sensing system can lead to a rapid, sensitive, low cost and miniaturizable device for the detection of PBDEs.
\end{abstract}

Keywords: Iridium Oxide Nanoparticles, Flame retardants, PBDEs, Electrocatalysis, Screen Printed Carbon Electrodes

\section{Introduction}

Polybrominated diphenyl ethers (PBDEs) have been used since 1970s as flame retardants in different products such as furniture, building materials or electronics (NOAA; Chem. Eng. News, 1971). However, it has been demonstrated that the exposure to these compounds causes severe health problems like neurodevelopmental deficits, thyroid homeostasis disruption, behavioral alteration, reproductive dysfunction and even cancer, reason why the use of PBDEs has been banned since 2004 in EU and USA (Guo et al., 2016; Linares et al., 2015; The Stockholm Convention, 2009; Ward et al., 2008). Nevertheless, PBDE molecules are hard to degrade and can persist long time bioaccumulated in mammalian organisms (in fat tissues) and in the environment, especially in marine water (Hooper and McDonald, 2000; Darnerud et al., 2001; Stapleton et al., 2008; Butryn et al., 2015; Chałupniak and Merkoçi, 2017). Among the different PBDEs structures, 3,3',4,4'-tetrabromodiphenyl ether (BDE-47) is one of the most abundant and resistant to degradation (Ahn et al., 2009; Li et al., 2014).

Although commercial kits for the detection of BDE-47 are available (e.g. Abraxis PBDE ELISA Kit), there is still a need of lower-cost, more portable, faster and more stable-in-time detection systems. The use of nanomaterials on sensing and biosensing is on the rise during last years (Riley, 2002; De la Escosura-Muñiz and Merkoçi, 2010, 2012; Quesada-González and Merkoçi, 2015, 2018; Robbs and Rees, 2016) due to the sensitivity improvement and robustness, among other properties, that nanoparticles can offer. In this work we have chosen iridium oxide (IV) nanoparticles $\left(\mathrm{IrO}_{2} \mathrm{NPs}\right)$ owing to their electrocatalytical properties towards water oxidation reaction (WOR) (Rivas et al., 2014), without requiring any other reagent. Screen printed carbon electrodes (SPCEs; Arduini et al., 2015; De la Escosura-Muñiz et al., 2011, 2016; Parolo et al., 2013; Talarico et al., 2015a, 2015b; Wang et al., 1998) are a suitable platform to carry on the reaction since they are easy to be fabricated and modified (both the design and the composition) also avoiding the fouling effect that occurs on classical electrodes since they are single-use ones (Dědík et al., 2011).

In this work we present a competitive electrochemical assay in which the measured current values (related to WOR, being catalyzed by the presence of $\mathrm{IrO}_{2} \mathrm{NPs}$ ) are inversely proportional to the concentration of BDE-47 in liquid sample. We take advantage of magnetic beads (MBs) coated with anti-PBDE antibodies to capture BDE-47 and horseradish peroxidase (HRP)-PBDE conjugate to link $\mathrm{IrO}_{2} \mathrm{NPs}_{\text {to }} \mathrm{PBDE}$ (HRP can be conjugated on $\mathrm{IrO}_{2} \mathrm{NPs}$ surface, as has been reported on other nanomaterials: Cui et al., 2008; Mohamed et al., 2017). Then, in absence of BDE-47, the conjugate MBs/PBDE-IrO ${ }_{2} \mathrm{NPs}$ will be formed, leading to a high electrocatalytic signal. In presence of free BDE-47 in the sample, the conjugate PBDE- $\mathrm{IrO}_{2} \mathrm{NPs}$ will be displaced, thus giving a decrease in the signal that is related with the amount of analyte as illustrated at Fig. 1. 


\section{Materials and methods}

\subsection{Materials}

BDE-47, bovine serum albumin (BSA), $\mathrm{K}_{2} \mathrm{IrCl}_{6}$, sodium citrate sesquihydrate, $\mathrm{NaOH}$ and phosphate buffer saline (PBS) tablets were purchased from Sigma-Aldrich. MBs coated with anti-PBDE antibody and PBDE-HRP conjugate were obtained from Abraxis PBDE ELISA Kit. The inks to fabricate the SPCEs (Electrodag 423SS carbon ink, Electrodag 6037SS Ag/AgCl ink, Minico 7000 blue insulating ink) were purchased from Acheson Industries and the substrate (Autostat HT5 polyester sheet) was purchased from McDermid Autotype.

\subsection{Instruments}

Allegra 64 R Centrifuge, Biosan TS-100 Thermoshaker, Malvern Zetasizer Nano 26, Autolab PGSTAT 302, hot plate Fisherbrand, Screen Printer DEK248, Tecnai F20 TEM (FEI), Perkin Elmer NexION 300X ICP-MS Spectrometer.

\subsection{Iridium oxide (IV) nanoparticles}

\subsubsection{Iridium oxide (IV) nanoparticles synthesis}

$\mathrm{IrO}_{2}$ NPs were synthesized following the procedure reported by Harriman and Thomas, 1987 and also previously applied in our group (Rivas et al., 2014, 2015; Mayorga-Martinez et al., 2014, 2015; Kurbanoglu et al., 2017). Briefly, a solution containing $1.24 \mathrm{mM} \mathrm{K}_{2} \mathrm{IrCl}_{6}$ and $3.80 \mathrm{mM}$ sodium citrate sesquihydrate, in MilliQ water was taken to $\mathrm{pH}$ 7.5 by using $0.25 \mathrm{M} \mathrm{NaOH}$. It was lead to ebullition in a reflux system for $30 \mathrm{~min}$ and the $\mathrm{pH}$ was checked after the solution was cooled down. If necessary, $\mathrm{pH}$ was readjusted to 7.5 and the 30 min ebullition step was repeated until $\mathrm{pH}$ was constant. Then, the solution was boiled for a last time during $2 \mathrm{~h}$ in presence of bubbling oxygen. The resulting solution was deep blue.

The solution of $\mathrm{IrO}_{2} \mathrm{NPs}$ was cleansed and concentrated 9 times by centrifuging it at $35000 \mathrm{rcf}$ and $4{ }^{\circ} \mathrm{C}$ during $2.5 \mathrm{~h}$, reconstituting the solution in a third part of its original volume with MilliQ water. To achieve the 9-fold concentration, the process was repeated twice.

Table 1. Z Potential measurements of $\mathrm{IrO}_{2} \mathrm{NPs}$ and the $\mathrm{IrO}_{2} \mathrm{NPs}-\mathrm{PBDE}$ conjugate at three different $\mathrm{pH}$.

pH $\quad \mathrm{IrO}_{2} \mathrm{NPs}-\mathrm{PBDE} \quad \mathrm{IrO}_{2} \mathrm{NPs}$

\begin{tabular}{lrr}
\hline $\mathbf{7}$ & $-42 \pm 2$ & $-54 \pm 2$ \\
$\mathbf{8}$ & $-45 \pm 3$ & $-55 \pm 1$ \\
$\mathbf{9}$ & $-49 \pm 1$ & $-55 \pm 2$
\end{tabular}

\subsubsection{Iridium oxide (IV) nanoparticles-PBDE conjugation}

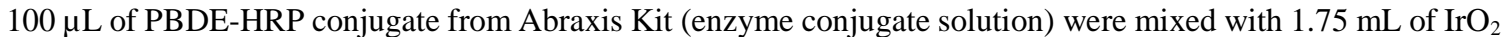
NPs during $2 \mathrm{~h}$ at $650 \mathrm{rpm}$ and room temperature. The mixture was left in repose overnight at room temperature and then centrifuged at $35000 \mathrm{rcf}$ and $4{ }^{\circ} \mathrm{C}$ during $2.5 \mathrm{~h}$. The precipitate was reconstituted in $1.85 \mathrm{~mL}$ of MilliQ water which $\mathrm{pH}$ was previously adjusted to 7.0.

\subsubsection{Iridium oxide (IV) nanoparticles characterization}

$\mathrm{IrO}_{2}$ NPs were characterized using transmission electron microscope (TEM) to evaluate their shape and homogeneity (Fig. 2). $\mathrm{Z}$ potential measurements at three different $\mathrm{pH}$ were carried out in order to verify the conjugation of $\mathrm{IrO}_{2}$ NPs and PBDE-HRP conjugate (Table 1 and Fig. S1). The concentration of $\mathrm{IrO}_{2} \mathrm{NPs}_{\text {, just }}$ synthetized and after centrifugation, was measured by inductively coupled plasma mass spectrometry (ICP-MS).

\subsection{Screen printed carbon electrodes fabrication}


A layer of carbon ink was printed onto a polyester sheet using a screen printer, forming the working and counter electrodes, later cured at $95{ }^{\circ} \mathrm{C}$ for $15 \mathrm{~min}$. Then, a second layer composed by $\mathrm{Ag} / \mathrm{AgCl}$ ink was printed for the reference electrode and was cured under the same conditions. Finally, an insulating ink was printed and cured for 20 min at $95^{\circ} \mathrm{C}$. Fig. S2 in the supporting information shows the design of the SPCE.

To evaluate the correct performance of the SPCE four different solutions were measured (by chronoamperometry, as explained on point 2.6): MBs, MBs incubated with $\mathrm{PBDE}-\mathrm{IrO}_{2} \mathrm{NPs}$ conjugate, $\mathrm{MBs}$ with $\mathrm{IrO}_{2}$ nanoparticles and MBs with PBDE.

\subsection{Assay preparation}

\subsubsection{Magnetic beads blocking}

$500 \mu \mathrm{L}$ of MBs with anti-PBDE antibody were incubated with $150 \mu \mathrm{L} \mathrm{5 \%} \mathrm{BSA} \mathrm{at} 650 \mathrm{rpm}$ and room temperature during $2 \mathrm{~h}$. The mixture was left in repose overnight at $4{ }^{\circ} \mathrm{C}$ and then washed twice with MilliQ water and twice with $0.1 \mathrm{M}$ PBS solution at $\mathrm{pH} 7.4$ using a magnetic rack. The solution was reconstituted in $500 \mu \mathrm{L}$ of the PBS solution.

\subsubsection{Samples preparation}

$500 \mu \mathrm{L}$ of blocked MBs were mixed with $250 \mu \mathrm{L}$ of sample solution (different concentrations of BDE-47 were evaluated, being MilliQ water used as blank) and incubated $20 \mathrm{~min}$ at room temperature and $650 \mathrm{rpm}$. Then, $250 \mu \mathrm{L}$ of the PBDE- $\mathrm{IrO}_{2}$ NPs conjugate were added and the incubation was repeated. The solution was washed in a magnetic rack and reconstituted in $250 \mu \mathrm{L}$ of PBS $0.1 \mathrm{M}$.

\subsection{Assay performance: $P B D E$ detection}

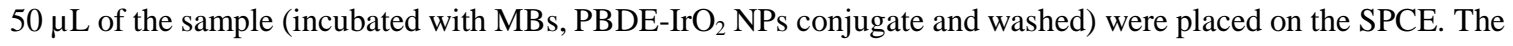
SPCE was placed over a magnet to ensure that the MBs are all deposited onto to the SPCE surface.

Following a previously reported procedure (Rivas et al., 2014), a fixed oxidative potential of $+1.3 \mathrm{~V}$ for $200 \mathrm{~s}$ was applied to achieve steady state current values. Hence, the current value at $200 \mathrm{~s}$ was considered as the analytical signal.

$50 \mu \mathrm{L}$ of concentrated $\mathrm{IrO}_{2}$ NPs were measured on the SPCE under the same conditions. This value was used to normalize the analytical signal by dividing the values obtained by this one.

\section{Results and discussion}

\subsection{Iridium oxide nanoparticles ( $\left.\mathrm{IrO}_{2} \mathrm{NPS}\right)$ characterization}

As seen on TEM image (Fig. 2), $\mathrm{IrO}_{2}$ NPs have an average size of $22 \pm 4 \mathrm{~nm}$.

$\mathrm{IrO}_{2} \mathrm{NPs}$ and $\mathrm{IrO}_{2}$ NPs-PBDE conjugate were stored $48 \mathrm{~h}$ at three different $\mathrm{pH}$ (7, 8 and 9). Then, aliquots before and after forming the conjugate were measured on $\mathrm{Z}$ potential obtaining the values shown on Table 1 . Due the absorption of PBDE on $\mathrm{IrO}_{2}$ NPs surface a variation in the charge is expected (Thielbeer et al., 2011) thus, since the highest variation was observed at $\mathrm{pH} 7$ it was chosen as optimal $\mathrm{pH}$ for the conjugate formation.

The concentration of $\mathrm{Ir}$ on $\mathrm{IrO}_{2}$ NPs should be around $1.24 \mathrm{mM}$ regarding the concentration of the precursor, $\mathrm{K}_{2} \mathrm{IrCl}_{6}$. According to the results obtained from ICP-MS (measuring ${ }^{193} \mathrm{Ir}$ isotope) the concentration was $1.26 \pm 0.08$ $\mathrm{mM}$ in an aliquot of just synthetized nanoparticles and $0.60 \pm 0.01 \mathrm{mM}$ after centrifugation. It indicates that during centrifugation nearly half of Ir is lost (does not precipitate), thus further concentration of the $\mathrm{IrO}_{2} \mathrm{NPs}_{\text {is necessary. }}$

\subsection{Sensing principle: specificity of the competitive assay}

MBs, MBs incubated with PBDE-IrO $\mathrm{IP}_{2}$ NP conjugate, $\mathrm{MBs}$ with $\mathrm{IrO}_{2}$ nanoparticles and $\mathrm{MBs}$ with PBDE solutions were washed on magnetic rack and measured on SPCE. At a potential of $+1.3 \mathrm{~V}$ it is expected that neither MBs nor PBDE produce high current signals, while $\mathrm{IrO}_{2}$ NPs should. On Fig. 3 it is observed how MBs and MBs with PBDE effectively barely produce analytical signal. In the case of $\mathrm{MBs}$ with $\mathrm{IrO}_{2} \mathrm{NPs}$, since there is no presence of PBDE both particles cannot be linked and, after the washing step on magnetic rack, $\mathrm{IrO}_{2} \mathrm{NPs}$ are removed also not generating

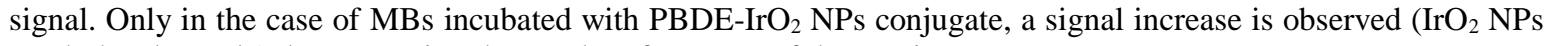
reach the electrode) demonstrating the good performance of the sensing system.

\subsection{PBDE detection}


Concentrations of 0 (blank; MilliQ water), 5, 25, 50 and 100 ppb of PBDE (BDE-47) were measured as explained at point 2.6 (and prepared as on point 2.5.2.). The results obtained are illustrated on Fig. 4, showing a good response of the current values related to the PBDE concentration in the sample since the current is decreased as higher is the concentration of PBDE, as expected from a competitive assay. The current response in a working range between 5 and $100 \mathrm{ppb}$ of PBDE (Fig. S3) follows the equation:

$$
\text { relative current }=-0.0351[\mathrm{PBDE}(\mathrm{ppb})]+9.1413
$$

With an $r$ value of 0.989 . The method shows a reproducibility (RSD) of $3 \%(n=3)$ for a PBDE concentration of 5 $\mathrm{ppb}$. A limit of detection (LOD) (calculated by dividing the average standard error of the measurements by the slope of the equation and then multiplying that value by 3.3; Hayashi et al., 2004) of $21.5 \mathrm{ppb}$ is estimated. Although our LOD is not the lowest reported for PBDE detection (Abraxis Kit has the lowest LOD reported, 0.02 ppb), our assay is, as far as we know, the first one for the detection of PBDE that does not require the use of enzymes, which makes our kit stable in time, cold storage independent and of high potential to be adapted for portability.

\section{Conclusion}

In this work we have demonstrated that $\mathrm{IrO}_{2}$ NPs can work as electrocatalytic tags for the detection of PBDEs in a competitive assay. Chronoamperometric measurements were performed obtaining higher current signal (related to water oxidation reaction) as higher was the amount of $\mathrm{IrO}_{2} \mathrm{NPs}$, opposed to the concentration of BDE-47, an abundant and not degradable type of PBDE. The LOD obtained was of $21.5 \mathrm{ppb}$.

Our system is a promising tool for fast and cheap measurement of PBDEs, avoiding the use of enzymes and of additional reagents, since the catalytic reaction occurs in aqueous buffer. Furthermore, $\mathrm{IrO}_{2} \mathrm{NPs}_{\text {are robust against }}$ temperature and stable in time. We believe that in the future it could easily become a miniaturized device, even coupled to a mobile phone (Quesada-González and Merkoçi, 2017).

\section{Acknowledgments}

We acknowledge support from EU (FP7, SMS project) and MINECO (Spain) MAT2017-87202-P. This work is also funded by the CERCA Programme / Generalitat de Catalunya. ICN2 is supported by the Severo Ochoa program from Spanish MINECO (Grant No. SEV-2013-0295). Daniel Quesada-González also acknowledges Autonomous University of Barcelona (UAB) for the possibility of performing this work inside the framework of Chemistry PhD Programme.

\section{References}

Abraxis PBDE ELISA Kit. Webpage: http://www.abraxiskits.com/moreinfo/PN500090info.pdf (last accessed: 24/06/2018)

Ahn, K. C., Gee, S. J., Tsai, H. J., Bennett, D., Nishioka, M. G., Blum, A., Fishman E., Hammock, B. D., 2009. Environ. Sci. Technol. 43, 7784-7790.

Arduini, F., Zanardi, C., Cinti, S., Terzi, F., Moscone, D., Palleschi, G., Seeber, R., 2015. Sens. Actuators B Chem. 212, 536-543.

Butryn, D., Gross, M., Chi, L. H., Schecter, A., Olson, J., Aga, D., 2015. Anal. Chim. Acta 892: 140-147.

Chałupniak, A., Merkoçi, A., 2017. Nano Res. 10 (7), 2296-2310.

Chem. Eng. News, 1971, 49 (43), 16-19, DOI: 10.1021/cen-v049n043.p016

Cui, R., Huang, H., Yin, Z., Gao, D., Zhu, J. J., 2008. Biosens. Bioelectron. 23 (11), 1666-1673.

Darnerud, P. O., Eriksen, G. S., Jóhannesson, T., Larsen, P. B., Viluksela, M., 2001. Environ. Health Perspect. 109 (1), 49-68.

De la Escosura-Muñiz, A., Merkoçi, A., 2010. Expert Opin. Med. Diagn. 4, 21-37.

De la Escosura-Muñiz, A., Parolo, C., Maran, F., Merkoçi, A., 2011. Nanoscale 3, 3350-3356.

De la Escosura-Muñiz, A., Merkoçi, A., 2012. G.I.T. Laboratory Journal Europe 16, 21-23.

De la Escosura-Muñiz, A., Baptista-Pires, L., Serrano, L., Altet, L., Francino, O., Sánchez, A., Merkoçi, A., 2016. Small 12 (2), 205-213.

Dědík, J., Janovcová, M., Dejmková, H., Barek, J., Pecková, K., 2011. Sensing in Electroanal. 6, 129-138.

Guo, W., Holden, A., Smith, S. C., Gephart, R., Petreas, M., Park, J. S., 2016. Chemosphere 150, 505-513.

Hayashi, Y., Matsuda, R., Maitani, T., 2004. Anal. Chem. 76, 1295-1301.

Harriman, A., Thomas, J. M., 1987. New J. Chem. 11, 757.

Hooper, K., McDonald, T. A., 2000. Environ. Health Perspect. 100 (5), 387-392.

Kurbanoglu, S., Rivas, L., Ozkan, S. A., Merkoçi, A., 2017. Biosens. Bioelectron. 88, 122-129.

Li, W., Sheng, P., Cai, J., Feng, H., Cai, Q., 2014. Biosens. Bioelectron. 61, 209-214. 
Linares, V., Bellés, M., Domingo, J. L., 2015. Arch. Toxicol. 89, 335-356.

Mayorga-Martinez, C. C., Pino, F., Kurbanoglu, S., Rivas, L., Ozkan, S. A., Merkoçi, A., 2014. J. Mater. Chem. B 2, 2233-2239.

Mayorga-Martinez, C. C., Chamorro-García, A., Serrano, L., Rivas, L., Quesada-González, D., Altet, L., Francino, O., Sánchez, A., Merkoçi, A., 2015. J. Mater. Chem. B 3, 5166-5171.

Mohamed, S. A., Al-Harbi, M. H., Almulaiky. Y. Q., Ibrahim, H. I., El-Shishtawy, R. M., 2017. Electron. J. Biotechnol. 27, 84-90.

NOAA (National Oceanic and Atmospheric Administration), U.S. Department of Commerce, What are PBDEs? Webpage: https://oceanservice.noaa.gov/facts/pbde.html (last accessed: 24/02/2018).

Parolo, C., Medina-Sánchez, M., Montón, H., de la Escosura-Muñiz, A., Merkoçi., A., 2013. Part. Part. Syst. Char. 30(8), 662-666.

Quesada-González, D., Merkoçi, A., 2015. Biosens. Bioelectron. 73, 47-63.

Quesada-González, D., Merkoçi, A., 2017. Biosens. Bioelectron. 92, 549-562.

Quesada-González, D., Merkoçi, A., 2018. Chem. Soc. Rev., DOI: 10.1039/C7CS00837F

Riley, D. J., 2002. Curr. Opin. Colloid Interface Sci. 7, 186-192.

Rivas, L., de la Escosura-Muñiz, A., Pons, J., Merkoçi, A., 2014. Electroanal. 26, 1287-1294.

Rivas, L., Mayorga-Martinez, C. C., Quesada-González, D., Zamora, A., de la Escosura-Muñiz, A., Merkoçi, A., 2015. Anal. Chem. 87 (10), 5167-5172.

Robbs, P. H., Rees, N. V., 2016. Phys. Chem. Chem. Phys. 18, 24812-24819.

Stapleton, H. M., Sjödin, A., Jones, R. S., Niehüser, S., Zhang, Y., Patterson Jr., D. G., 2008. Environ. Sci. Technol. 42, 3453-3458.

Talarico, D., Cinti, S., Arduini, F., Amine, A., Moscone, D., Palleschi, G., 2015a. Environ. Sci. Technol. 49 (13), 7934-7939.

Talarico, D., Arduini, F., Amine, A., Moscone, D., Palleschi, G., 2015b. Talanta 15, 267-272.

The Stockholm Convention, $2009 . \quad$ Webpage:

http://chm.pops.int/TheConvention/Overview/TextoftheConvention/tabid/2232/Default.aspx\#LiveContent[convtext

] (last accessed: 24/06/2018).

Thielbeer, F., Donaldson, K., Bradley, M., 2011. Bioconjugate Chem. 22, 144-150.

Wang, J., Tian, B., Nascimento, V. B., Angnes, L., 1998. Electrochimica Acta 43 (23), 3459-3465.

Ward, J., Mohapatra, S. P., Mitchell, A., 2008. Environ. Int. 34, 1148-1156.

\section{Supplementary Material}

Supplementary material can be found at: https://www.journals.elsevier.com/biosensors-andbioelectronics/XXXX 


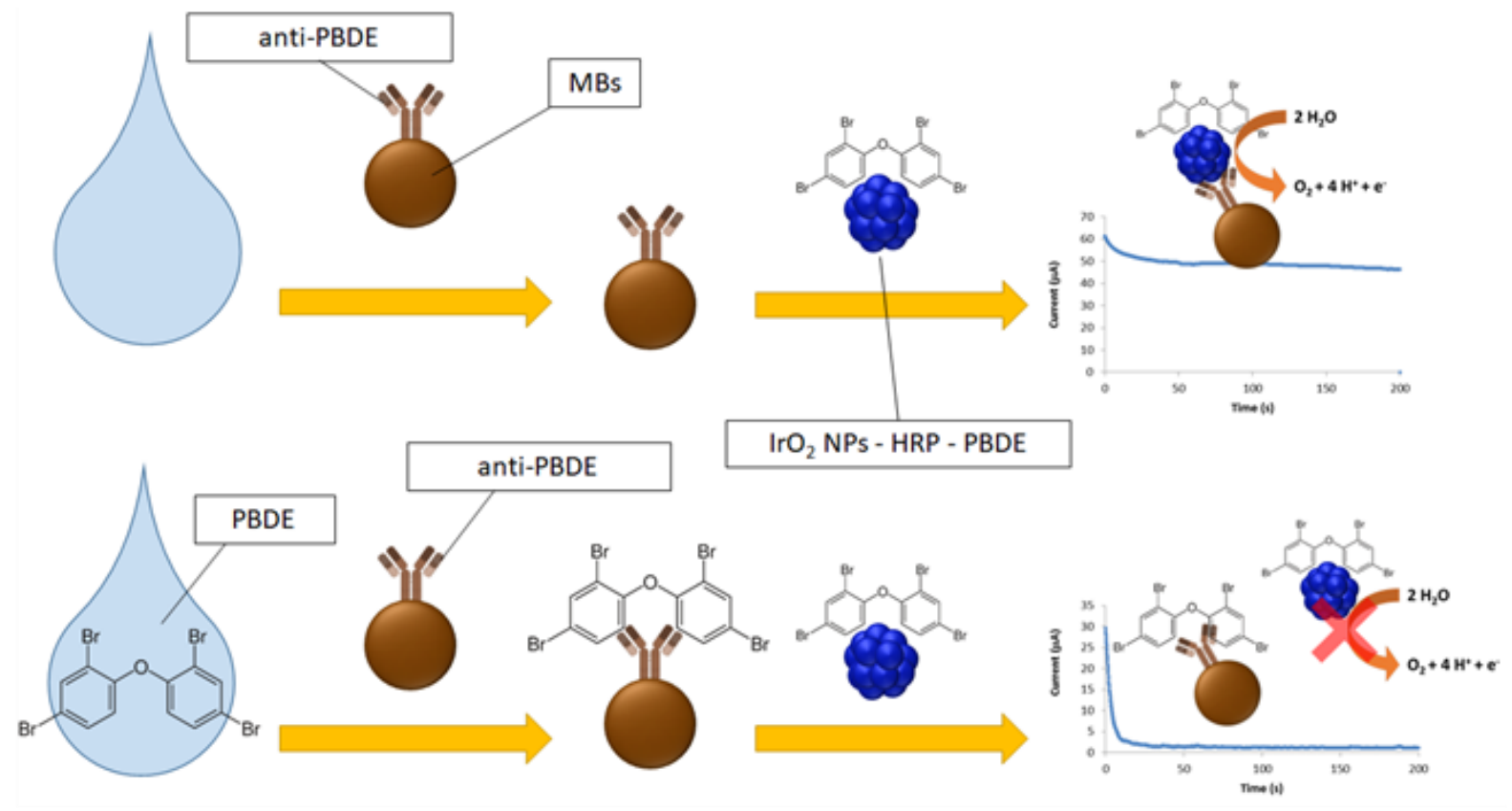

Figure 1. Schematic representation of the competitive electrocatalytic assay. In absence of PBDE, PBDE-IrO_ $\mathrm{NPs}_{2}$ conjugate is captured by MBs producing a current increase due the catalysis of WOR (up). In presence of PBDE, IrO2 NPs are not captured and signal is inhibited (down).

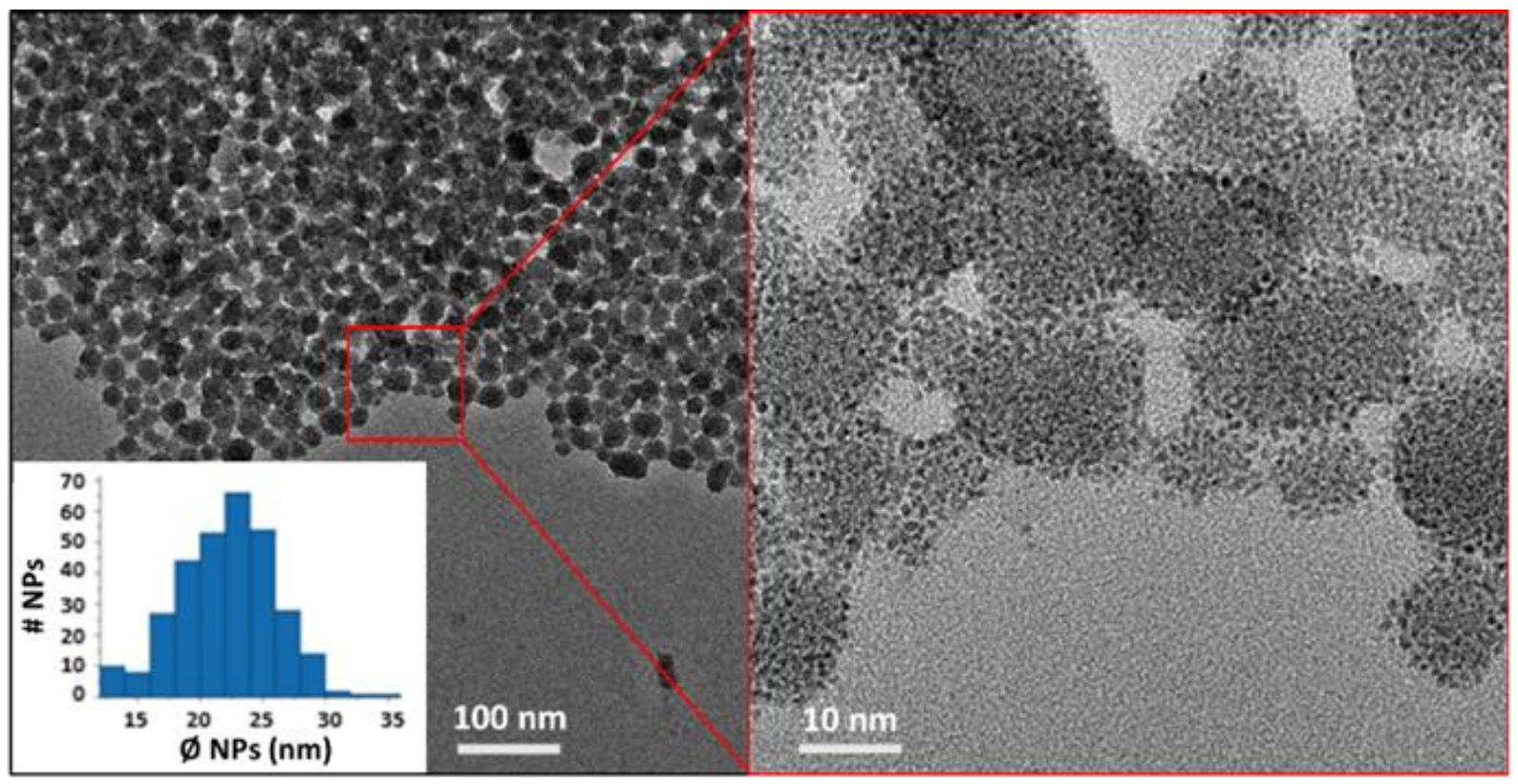

Figure 1. TEM images of $\mathrm{IrO}_{2} \mathrm{NPs}$ with the corresponding histogram (inset) (average diameter $22 \pm 4 \mathrm{~nm}$ ). 


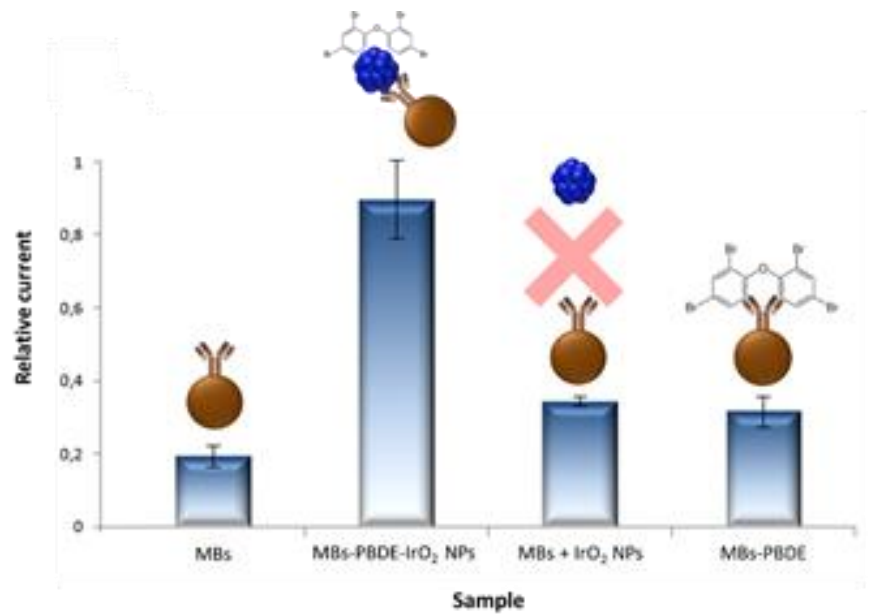

Figure 1. Relative current measurements on SPCE for MBs, MBs and $\mathrm{PBDE}-\mathrm{IrO}_{2} \mathrm{NPs}$ conjugate, $\mathrm{MBs}$ and $\mathrm{IrO}_{2} \mathrm{NPS}, \mathrm{MBs}$ and $\mathrm{PBDE}$.

a)

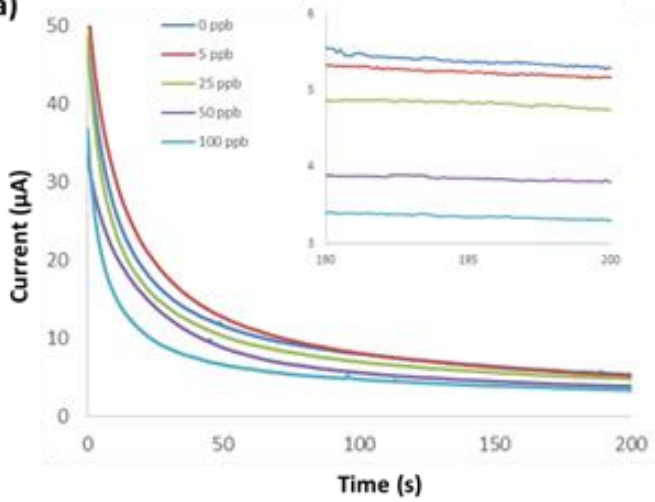

b)

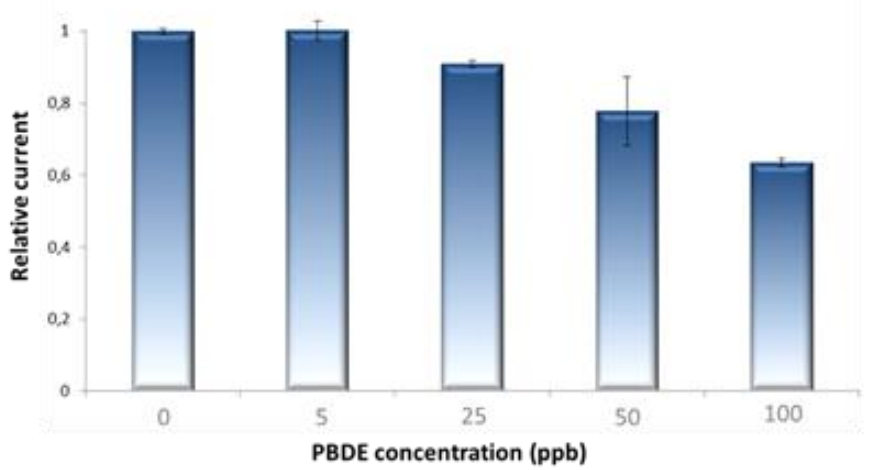

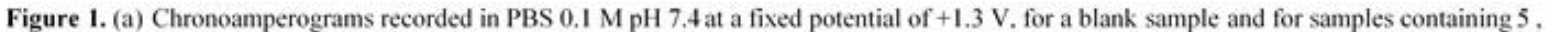
25,50 and $100 \mathrm{ppb}$ of PBDE (inset graph details the currents recorded at 200 seconds, which represent the analytical signal). (b) Corresponding relative current measurements PBDE concentrations. Samples correspond to the competitive assay detailed at the experimental section 